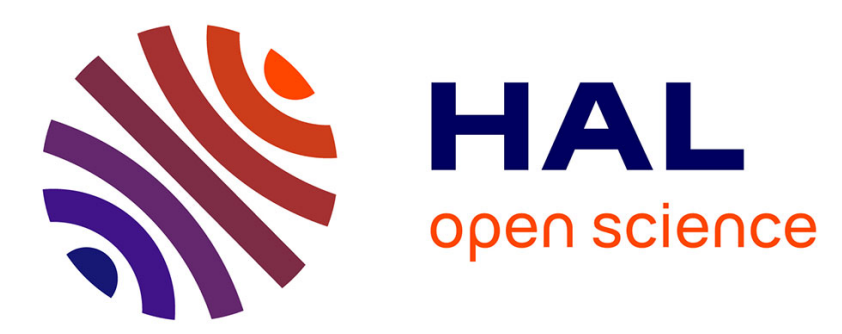

\title{
Macroscopic Model of Two-Phase Compressible Flow in Double Porosity Media
}

\author{
Mikhail Panfilov, Zh. Baishemirov, A. Berdyshev
}

\section{To cite this version:}

Mikhail Panfilov, Zh. Baishemirov, A. Berdyshev. Macroscopic Model of Two-Phase Compressible Flow in Double Porosity Media. Fluid Dynamics / Izvestiya Akademii Nauk - Mekhanika Zhidkosti i Gaza, 2020, 55 (7), pp.936-951. 10.1134/s001546282007006x . hal-03264614

\section{HAL Id: hal-03264614 \\ https://hal.univ-lorraine.fr/hal-03264614}

Submitted on 18 Jun 2021

HAL is a multi-disciplinary open access archive for the deposit and dissemination of scientific research documents, whether they are published or not. The documents may come from teaching and research institutions in France or abroad, or from public or private research centers.
L'archive ouverte pluridisciplinaire HAL, est destinée au dépôt et à la diffusion de documents scientifiques de niveau recherche, publiés ou non, émanant des établissements d'enseignement et de recherche français ou étrangers, des laboratoires publics ou privés. 
Panfilov M., Baishemirov Zh.D., and Berdyshev A.S.

Macroscopic Model of Two-phase Compressible Flow in Double Porosity Media.

Fluid Dynamics, 2020, Vol. 55, no. 7, pp. 936-951.

\title{
MACROSCOPIC MODEL OF TWO-PHASE COMPRESSIBLE FLOW IN DOUBLE POROSITY MEDIA
}

\author{
M. Panfilov ${ }^{1,2 *}$, Zh.D. Baishemirov ${ }^{3,4}$, and A.S. Berdyshev ${ }^{3,4}$ \\ ${ }^{1}$ Institut Elie Cartan - Université de Lorraine, France \\ ${ }^{2}$ Institut d'Alembert - Sorbonne Université, France \\ ${ }^{3}$ Kazakh National Pedagogical University of Abay, \\ Almaty, Kazakhstan \\ ${ }^{4}$ UInstitut of informatic and computational technologies, \\ Almaty, Kazakhstan \\ *e-mail: michel.panfilov@univ-lorraine.fr
}

Received 27.06.2019

After revision 20.09.2019

Accepted to publication 13.11.2019

A macroscopic model of two-phase flow of compressible liquids in a compressible double porosity medium is developed and used to analyse various qualitative mechanisms of the occurrence of memory (delay). The two main mechanisms are non-instantaneous capillary redistribution of liquids, and non-instantaneous relaxation of pressure. In addition, cross effects of memory arise, caused by asymmetric extrusion of liquids from pores due to phase expansion and pore compaction, as well as nonlinear overlap of compressibility and capillarity (non-linear extrusion). To construct the model, the 
asymptotic method of two-scale averaging in the variational formulation is applied. Complete averaging has been achieved due to the separation of nonlocality and nonlinearity at different levels of the asymptotic expansion. All delay times are explicitly defined as functions of saturation and pressure.

Key words: double porosity, averaging, two-phase flow, compressible fluid, nonlocality, memory, delay, nonequilibrium

Introduction. A double porosity medium (it is also called fractured-porous medium) is a classical model for studying the effects of the occurrence of memory in fluid flow after changing the scale of consideration. Such a medium consists of weakly permeable blocks and highly permeable fractures. In the case of a single-phase compressible flow, the propagation of a perturbation in blocks is much slower than in fractures, so that the average pressure in the blocks is delayed and depends on the entire history of the variation of the average pressure in the fractures, which is a memory effect. Since this effect is observed in terms of average pressures, it is necessary to construct equations averaged over inhomogeneities. For single-phase flow, which is described by linear equations, it is possible to obtain a completely averaged model and explicit expression for the memory operator. The first mathematical model of such a process was proposed in [9]. Later, in the early 1990s, it was shown by averaging [7, 20] that high difference in the permeability of blocks and fractures leads not only to delay, but to long memory, described by an integro-differential equation.

In the case of two-phase flow, another type of memory arises, which is generated by capillarity. Capillary forces push the wetting phase from the fractures into blocks, while displacing the non-wetting phase from the blocks into fractures. This phenomenon is known as capillary countercurrent imbibition. In blocks, it is slower than in fractures, which causes a delay in the behavior of phase saturation ("saturation" means the volume fraction of the phase in the pores). The dynamics of saturation waves is nonlinear, proper to any two-phase flows. Therefore, a situation arises when the 
delay/memory at the macroscale is caused by the propagation of a nonlinear wave on the local scale. For brevity, such a memory will be called nonlinear. If the two phases are compressible, then two types of memory arise, caused by compressibility and capillarity, which can interact between them while leading to new physical effects. The discovery and analysis of these physical effects is the purpose of this work. For this, it was first necessary to develop a macroscopic model of two-phase flow of compressible liquids in a double porosity medium.

Attempts to construct such models were carried out mainly for incompressible systems $[3,4,6,10,11,20,24]$. However, the occurrence of nonlinear memory is a strong obstacle and leads to the fact that macroscopic model is not completely averaged. This means that the macroscopic equations and the local problem are not split, but constitute a single entangled system of equations that must be solved together and repeatedly for each macroscopic point in space and every moment in time. Since such a result is not simpler than the initial unaveraged problem, its significance for physics is very problematic. Therefore, the problem of constructing an averaged model remains open even for incompressible fluids. Attempts to overcome it by linearizing the capillary imbibition equations, as was done in $[4,8]$, physically mean that the flow in the blocks is replaced by a single-phase flow.

The authors are aware of two articles devoted to the two-phase flow of compressible fluids in a double porosity medium [1,5]. In [5], one phase was a compressible gas, and the second incompressible, and in [1] both phases are compressible. Mathematical advances have been made in the field of proving the convergence of the original system to the averaged one, under the condition of the interaction between the nonlocality, double nonlinearity, and double degeneracy (parabolic equations), however, the obtained models remain incompletely averaged.

In this regard, the physics of two-phase flows in fractured-porous media continues to be studied not only by averaging methods, but also by other methods. Pore-level experimental studies are carried out on glass micromodels [14, 15, 23] with visual observation of the process. Experiments at the Darcy level are conducted in opaque 
rock samples scanned by nuclear magnetic resonance [16]. Numerical simulation at the pore level, based on the Navier-Stokes equations, uses the Cahn - Hilliard diffuse interface method [13,21,23], which makes it possible to track the interface and its deformation. Numerical modeling based on Darcy's law is currently focused primarily on the problem of coupling a three-dimensional problem in a block and a twodimensional problem in a flat fracture $[12,22]$. In all these studies, similar results were obtained regarding the role of capillary imbibition on oil recovery: the greater the capillary pressure, the more intensive the imbibition and the higher amount of oil is produced from the blocks. However, there are conflicting results regarding the effect of the displacement rate on oil recovery: in [13, 15, 21, 22], the recovery decreases with increasing injection rate, while in $[14,16]$ it increases. This indicates that the general physical theory of two-phase flows in a medium with strong inhomogeneities remains an open question. In the present paper, we propose a theoretical approach that allows us to construct a completely averaged model of such systems, despite the occurrence of nonlinear memory in them. It combines the elements of a single-phase compressible and two-phase incompressible systems. It is shown that, in addition to the capillary delay of saturation and the delay of pressure due to compressibility, two new cross effects also arise: the effect of delayed saturation caused by the asymmetric extrusion of phases from blocks due to phase expansion and pore compaction; and the effect of delayed saturation caused by a nonlinear overlap of compressibility and capillarity.

We applied the homogenization technique for the two-scale formulation of the problem in a variational form, similar to the constructive technique of the two-scale convergence method [2]. It enables us to carry out all the calculations in the most compact form. It should be emphasized that the compressibility in our work concerns primarily the fluids, while the compressibility of the porous medium is considered to be weak. The medium compressibility means that it changes the pore volume depending on the pressure of the fluid filling it (such a deformation of the medium is called the compaction). Fully deformable media are not considered here. Approaches 
to averaging deformable elastic saturated porous media are described, for example, in $[17,18]$.

\section{Problem setting}

1.1 Equations of two-phase flow of compressible fluids. Compressibility means that fluid densities and porosity/permeability of porous medium are pressure dependent. Traditionally, the compressibility of porous media is considered to be weak and is described by a linear law, while the compressibility of liquids is described by a more general exponential law [19]:

$$
\frac{d \rho_{w}}{d p}=\tilde{\beta}_{w} \rho_{w}, \quad \frac{d \rho_{o}}{d p}=\tilde{\beta}_{o} \rho_{o}, \quad \frac{d \phi}{d p}=\tilde{\beta}_{\phi} \phi^{0}
$$

where $p$ is the pressure, $\phi^{0}$ is a characteristic value of the porosity; $\tilde{\beta}_{w}, \tilde{\beta}_{o}, \tilde{\beta}_{\phi}$ are the isothermal coefficients of compressibility that are accepted to be constant. Their dimension is $\mathrm{Pa}^{-1}$. For water, oil and geological rocks, the value of a compressibility coefficient is $10^{-9}-10^{-8} \mathrm{~Pa}^{-1}$. A positive derivative $d \phi / d p$ means that the porosity decreases if the fluid pressure in the pores decreases. This corresponds to the pore compaction under the weight of overlying rocks.

For single-phase compressible fluids in a deformable medium, the classical simplification of flow equations is used, which is as follows. We substitute the compressibility equations (1.1) into the mass conservation equation: $\partial_{t}(\phi \rho)+$ $\nabla \cdot(\rho \mathbf{V})=0$, where $\mathbf{V}$ is the Darcy velocity. Then we obtain: $0=(\phi \rho)^{\prime} \partial_{t} p+\rho \nabla \cdot \mathbf{V}+\rho^{\prime} \mathbf{V}$. $\nabla p$, where «prime» means the derivative with respect to pressure. The last term, $\mathbf{V} \cdot \nabla p \sim$ $(\nabla p)^{2}$, is negligible, as the gradients are very low in underground porous media. 
Then we obtain the classical equation of single-phase flow of compressible fluids in deformable rocks:

$$
\phi^{0} \beta \partial_{t} p+\nabla \cdot \mathbf{V}=0 \text {, where } \beta=\frac{(\phi \rho)^{\prime}}{\phi^{0} \rho}=\tilde{\beta}_{w}+\tilde{\beta}_{\phi}
$$

One accepts that the Darcy velocity $\mathbf{V}$ is proportional to the absolute permeability $K$.

Further, the porosity $\phi^{0}$ and permeability $K$ are considered to be constant.

The same assumption can be applied to two-phase flow. Consider immiscible phases and call them water and oil. Phases have different pressures: $p_{w}$ and $p_{o}$. That is why $\rho_{w}=\rho_{w}\left(p_{w}\right)$ и $\rho_{o}=\rho_{o}\left(p_{o}\right)$. The classical equations of the theory of two-phase flow in porous media are applicable for the channel structure of flow [25] when each phase occupies its own pores. Therefore, at every moment of time, $\phi=\phi\left(p_{w}\right)$ for pores occupied with water, and $\phi=\phi\left(p_{o}\right)$ for pores occupied with oil. The initial equations of mass conservation of both phases have the form:

$$
\partial_{t}\left(\phi \rho_{w} s\right)+\nabla \cdot\left(\rho_{w} \mathbf{V}_{w}\right)=0, \text { and } \quad \partial_{t}\left(\phi \rho_{o}(1-s)\right)+\nabla \cdot\left(\rho_{o} \mathbf{V}_{o}\right)=0
$$

where $s$ is the volume fraction of water in pores (the saturation), $\rho$ is the phase density, $\phi$ is the medium porosity, $p$ is the pressure, $\mathbf{V}$ is the vector of Darcy velocity. The idexes $w$ and $o$ mean water and oil. Differentiating by parts, we obtain for water:

$$
\phi \rho_{w} \partial_{t} s+\frac{d\left(\phi \rho_{w}\right)}{d p_{w}} s \partial_{t} p_{w}+\rho_{w} \nabla \cdot \mathbf{V}_{w}+\frac{d \rho_{w}}{d p_{w}} \nabla p_{w} \cdot \mathbf{V}_{w}=0
$$


Neglecting the terms of the order $(\nabla p)^{2}$, we finally obtain the system of equations for the two-phase flow of compressible fluids in a weakly compressible porous medium:

$$
\left\{\begin{array}{l}
\phi \partial_{t} s+\phi \beta_{w} s \partial_{t} p_{w}+\nabla \cdot \mathbf{V}_{w}=0, \\
-\phi \partial_{t} s+\phi \beta_{o}(1-s) \partial_{t} p_{o}+\nabla \cdot \mathbf{V}_{o}=0
\end{array}\right.
$$

where $\beta_{w} \equiv \tilde{\beta}_{w}+\tilde{\beta}_{\phi}, \beta_{o} \equiv \tilde{\beta}_{o}+\tilde{\beta}_{\phi}$, and the value $\phi^{0}$ is denoted simply as $\phi$.

This system is supplemented by the equations of conservation of momentum in the form of Darcy's law for each phase and the equation of capillary equilibrium, which relates the pressure in the phases:

$$
\begin{gathered}
\mathbf{V}_{\alpha}=-K \lambda_{\alpha} \nabla p_{\alpha}, \quad \alpha=w, o ; \quad \lambda_{\alpha}(x, s) \equiv \frac{k_{\alpha}(x, s)}{\mu_{\alpha}} \\
p_{o}=p_{w}+p_{c}(s)
\end{gathered}
$$

where $K$ is the absolute permeability of the medium, $\mu$ is the dynamic viscosity. We emphasize that $K$ and $\phi$ are considered to be independent of pressure, which corresponds to a weakly compressible porous medium. The relative permeability $k_{w}(s), k_{o}(s)$ and capillary pressure $p_{c}(s)$ are given and have the following properties:

- $k_{w}(s)$ is a continuous and monotonically non-decreasing function of water saturation such that $k_{w} \equiv 0$ for $s \in\left[0, s_{*}\right]$, and $k_{w}(1)=1$;

- $k_{o}(s)$ is a continuous and monotonically non-increasing function such that $k_{o}(0)=1$ and $k_{o} \equiv 0$ for $s \in\left[s^{*}, 1\right]$; 
- $p_{c}(s)$ is a continuous and monotonically non-increasing function such that $p_{c}(1)=0$ and $p_{c} \rightarrow \infty$ for $s \rightarrow s_{*}$. It is not defined for $s \in\left[0, s_{*}\right]$.

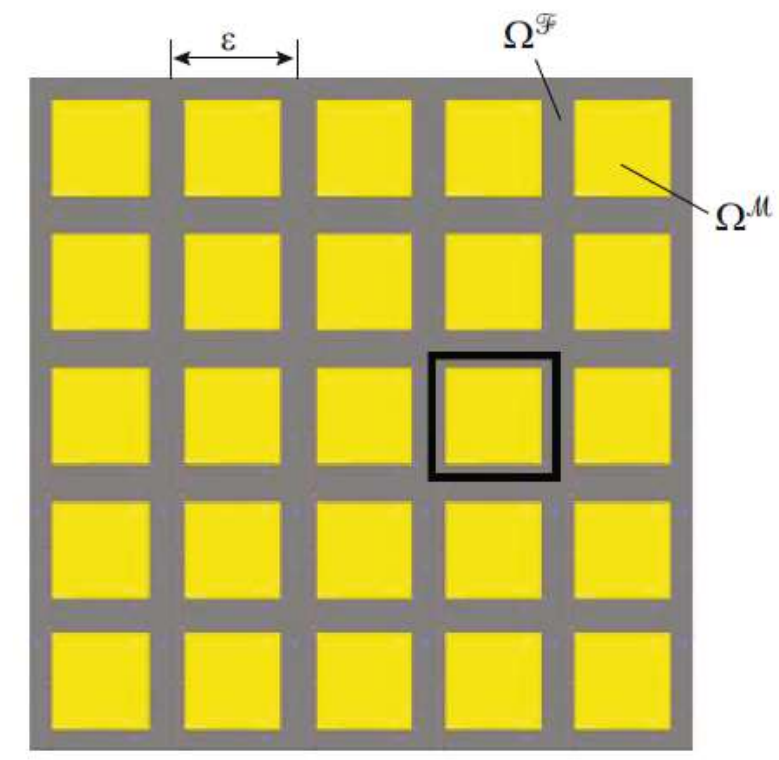

Fig. 1. Medium geometry.

1.2 Problem of homogenization. Let the reservoir $\Omega \subset \mathfrak{R}^{d}(d=2,3)$ be a bounded, connected region having a periodic microstructure with a characteristic scale $\varepsilon$, which is the ratio of the heterogeneity period to the length of the entire region. Parameter $\varepsilon$ is small: $0<\varepsilon \ll 1$ (Fig. 1). The unit cell of this microstructure is marked by a black square.

Being stretched $\varepsilon^{-1}$ times along each axis, it forms a cube $Y=(0,1)^{d}$ with side 1 , where $d$ is the space dimension. This cube consists of two subdomains: a connected subdomain $Y^{\mathcal{F}}$ ("fractures") and $Y^{\mathcal{M}}$ ("matrix block"). Denote by $\Gamma$ the interface between $Y^{\mathcal{F}}$ and $Y^{\mathcal{M}}$ in $Y$.

Four equations of mass and momentum conservation (1.2), (1.3) can be reduced to two equations eliminating the flow velocity: 


$$
\phi \partial_{t} s_{\alpha}+\phi s_{\alpha} \beta_{\alpha} \partial_{t} p_{\alpha}=\nabla \cdot\left(K \lambda_{\alpha} \nabla p_{\alpha}\right), \quad \alpha=w, o
$$

where $s \equiv s_{w}, \quad s_{o}=1-s_{w}$.

System (1.5) and (1.4) determines three unknown functions: water saturation $s$, pressure in water $p_{w}$ and pressure in oil $p_{o}$.

At the interface $\Gamma$, the normal fluxes of each phase and phase pressures are continuous.

The boundary conditions specify the water pressure and the saturation. The initial conditions fixe a given distribution of saturation and pressure in the domain:

$$
s(x, 0)=s^{0}(x), \quad p_{w}(x, 0)=p_{w}^{0}(x)
$$

1.3 Parameters of the problem. Porosity, compressibility, phase permeability, and capillary pressure are different in blocks and cracks, but are of the same order of magnitude:

$$
\begin{gathered}
\phi(x)=\left\{\begin{array}{l}
\phi^{\mathcal{F}} \\
\phi^{\mathcal{M}},
\end{array} \quad \beta_{\alpha}(x)= \begin{cases}\beta_{\alpha}^{\mathcal{F}}, & x \in \Omega^{\mathcal{F}} \\
\beta_{\alpha}^{\mathcal{M}}, & x \in \Omega^{\mathcal{M}}\end{cases} \right. \\
\lambda_{\alpha}(s, x)=\left\{\begin{array}{l}
\lambda_{\alpha}^{\mathcal{F}}(s) \\
\lambda_{\alpha}^{\mathcal{M}}(s),
\end{array} \quad p_{c}(s, x)=\left\{\begin{array}{ll}
p_{c}^{\mathcal{F}}(s), & x \in \Omega^{\mathcal{F}} \\
p_{c}^{\mathcal{M}}(s), & x \in \Omega^{\mathcal{M}},
\end{array} \quad \alpha=w, o ;\right.\right.
\end{gathered}
$$

where $\phi^{\mathcal{F}}, \phi^{\mathcal{M}}, \beta_{\alpha}^{\mathcal{F}}, \beta_{\alpha}^{\mathcal{M}}, \lambda_{\alpha}^{\mathcal{F}}(s), \lambda_{\alpha}^{\mathcal{M}}(s), p_{c}^{\mathcal{F}}(s), p_{c}^{\mathcal{M}}(s)$ are independent of $\varepsilon$. Parameters $\phi^{\mathcal{F}}, \phi^{\mathcal{M}}, \beta_{\alpha}^{\mathcal{F}}, \beta_{\alpha}^{\mathcal{M}}$ are positive. Functions $\lambda_{\alpha}^{\mathcal{F}}(s), \lambda_{\alpha}^{\mathcal{M}}(s), p_{c}^{\mathcal{F}}(s), p_{c}^{\mathcal{M}}(s)$ are 
non-negative. We assume that $p_{c}^{\mathcal{M}}(s) \geq p_{c}^{\mathcal{F}}(s)$ since the capillary pressure is greater where permeability is less.

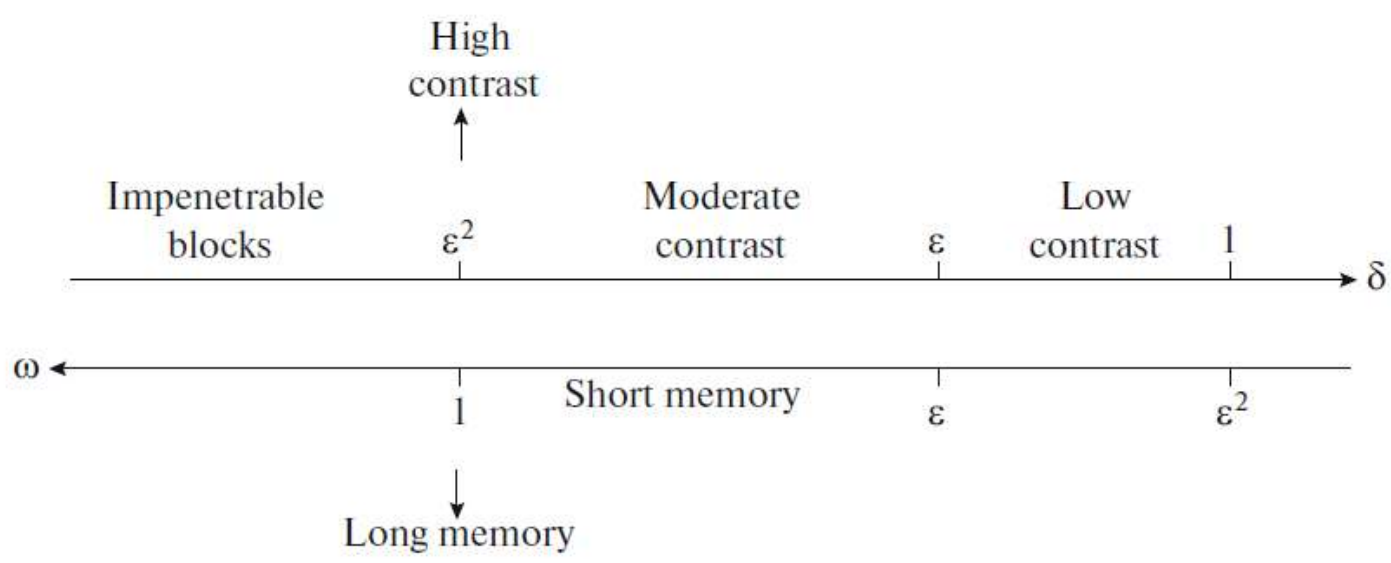

Fig. 2. Classification of porous media with respect to parameters of contrast $(\delta)$ and the memory length $(\omega)$.

At the same time, the absolute permeability of blocks and fractures can differ very significantly. It is known that when the porosity varies within $0.1-0.2$, the permeability changes by several orders of magnitude. Therefore, we accept the basic condition of double porosity:

$$
K(x)=\left\{\begin{array}{l}
K^{\mathcal{F}}, \quad x \in \Omega^{\mathcal{F}} \\
\delta K^{\mathcal{M}}, \quad x \in \Omega^{\mathcal{M}}
\end{array}\right.
$$

where parameter $\delta$ is small. Coefficients $K^{\mathcal{M}}$ and $K^{\mathcal{F}}$ are positive and independent of $\varepsilon$.

Parameter $\delta>0$ is the degree of contrast between the permeability of blocks and fractures. Great contrast causes delay or memory. The degree of delay (or "the memory length") is expressed by another parameter, which is defined as follows: 


$$
\omega=\frac{\varepsilon^{2}}{\delta}
$$

It arises in the problem in an explicit form if the initial equations (1.5) are written not through macroscopic variables $x_{i}$, but are reformulated in terms of microscopic coordinates $y_{i}=x_{i} / \varepsilon$. Then the right-hand side of equations (1.5) will take the following form in the block: ... $=\omega^{-1} \nabla_{y} \cdot\left(K^{\mathcal{M}} \lambda_{\alpha} \nabla_{y} p_{\alpha}\right)$

In terms of contrast and memory length, two types of media are of interest: - media with a long memory: $\omega \sim 1$, or with strong contrast: $\delta \sim \varepsilon^{2}$. They are also called $\varepsilon^{2}$-media;

- media with short memory: $\varepsilon \ll \omega \ll 1$, or with moderate contrast: $\varepsilon^{2} \ll \delta \ll \varepsilon$.

The classification of media by parameters $\delta$ and $\omega$ is given in Fig. 2.

There are also non-contrast media: $\delta \sim 1$, low-contrast $\varepsilon \ll \delta \ll 1$, and media with impermeable blocks: $\delta \ll \varepsilon^{2}$. In the first and second case, the exchange process between blocks and fractures occurs without delay, and in the third case, it is completely absent due to a very strong delay; therefore, all these three classes are not of interest for the purposes of our study.

2. Method of homogenization with splitting the non-locality and nonlinearity 
2.1 Splitting nonlocality and nonlinearity. The purpose of this paper is to obtain a fully averaged model. This concept was introduced in [4]. A completely averaged model is such that the macroscopic equations do not contain microscopic variables, and the cell problem, which determines the effective coefficients, is independent of macroscopic variables and therefore is solved only once.

To overcome the overlap of nonlinearity and nonlocality described earlier, we will separate them to different levels of asymptotic expansion over the memory length parameter $\omega$. In other words, nonlinearity is preserved in the zero order approximation, while nonlocality is in the first order. Since the first approximation of an asymptotic expansion usually leads to linear problems, we expect to describe nonlocality explicitly and completely. For incompressible liquids, this method was applied in [11].

Thus, parameter $\omega$ must be small, that is, we should analyze media with moderate contrast between the permeabilities of blocks and fractures. In the general case, we are forced to work with two-parameter asymptotic expansions: over the period of heterogeneity $\varepsilon$ and over the parameter of the memory length $\omega$, which are independent. Given the ordering shown in Fig. 2, the asymptotic expansion of any function $f$ is as follows:

$$
f^{\varepsilon, \omega}(x, t)=\sum_{k=0}^{\infty} \sum_{j=0}^{\infty} \omega^{k} \varepsilon^{j} f_{k j}^{\varepsilon}(x, t)=f_{00}+\omega f_{01}^{\varepsilon}+\varepsilon f_{10}^{\varepsilon}+\ldots
$$

where the superscripts $\varepsilon$ and $\omega$ mean the dependence on the corresponding parameters. Moreover, it was taken into account that the problem is regular in $\omega$ (except small 
times $t \sim \omega$ after the perturbation) and is regularly perturbed in $\varepsilon$, therefore, the coefficients $f_{k j}^{\varepsilon}$ depend on $\varepsilon$ and do not depend on $\omega$. If $\varepsilon \rightarrow 0$, then the inhomogeneities tend to points, that is, the medium transforms into a homogeneous averaged one. If $\omega \rightarrow 0$, then the memory disappears in the system. Therefore, the zeroth approximation $f_{00}$ corresponds to the averaged value of the function in the system without contrast between the blocks and fractures.

To build an averaged model, the zeroth and first approximations are enough: $f_{00}, f_{01}^{\varepsilon}$ , $f_{10}^{\varepsilon}$. In this case, the term $\omega f_{01}^{\varepsilon}$ describes the contribution of the contrast in properties, that is, memory effects, while $\varepsilon f_{10}^{\varepsilon}$ reflects the contribution of spatial oscillations of permeability. Obviously, the averaging technique is greatly simplified if we choose $\omega$ so that, for example:

$$
\omega=\sqrt{\varepsilon}, \text { or } \delta=\varepsilon^{3 / 2}
$$

which was used in [11].

Then the complete asymptotic expansion reduces to a one-parametric expansion in $\sqrt{\varepsilon}$

2.2 Two-scale formulation of the problem. First of all, we introduce an extension of the solution $\tilde{s}(x, y, t), \tilde{p}(x, y, t)$, so that

$$
p_{w}(x, t)=\left.\tilde{p}(x, y, t)\right|_{y=\frac{x}{\varepsilon}}, \quad s(x, t)=\left.\tilde{s}(x, y, t)\right|_{y=\frac{x}{\varepsilon}}
$$


Then the following is true for derivatives:

$$
\left.\frac{\partial p(x, t)}{\partial x_{i}} \rightarrow\left(\frac{\partial \tilde{p}(x, y, t)}{\partial x_{i}}+\frac{1}{\varepsilon} \frac{\partial \tilde{p}(x, y, t)}{\partial y_{i}}\right)\right|_{y=\frac{x}{\varepsilon}}
$$

Since the operations of extension and differentiation commute, we see that the argument $y$ can be considered as an independent variable from $x$, but we should set it equal to $x / \varepsilon$ in the final results. The two-scale formulation of equations (1.5) has the following form:

$$
\left\{\begin{array}{l}
\phi(y) \partial_{t} s_{\alpha}+\phi(y) \beta_{\alpha}(y) s_{\alpha} \partial_{t} p_{\alpha}=\left(\partial_{x i}+\frac{1}{\varepsilon} \partial_{y i}\right) \cdot\left(K(y) \lambda_{\alpha}\left(\partial_{x i} p_{\alpha}+\frac{1}{\varepsilon} \partial_{y i} p_{\alpha}\right)\right), \quad x \in \Omega, \quad y \in Y, \quad t \in(0, T) \\
p_{o}=p_{w}+p_{c}, \quad s_{o}=1-s_{w}
\end{array}\right.
$$

Hereinafter, the repeating indices $i, k, j$ mean the sum from 1 to 3 . With conditions:

$$
\left\{\begin{array}{l}
\left.s_{w}\right|_{t=0}=s^{0}(x),\left.\quad p_{w}\right|_{t=0}=p_{w}^{0}(x) \\
{\left[K \lambda_{\alpha} \frac{\partial p_{\alpha}}{\partial n}\right]_{\Gamma}=0, \quad\left[p_{\alpha}\right]_{\Gamma}=0, \quad \alpha=w, o} \\
s_{w}, p_{w} \text { 1-periodic in } y
\end{array}\right.
$$

and Neumann or Dirichlet conditions at the boundary of the domain, $\partial \Omega$. Here $[\cdot]$ is the jump symbol.

It can be represented in a variational formulation: 


$$
\begin{aligned}
& \iint_{\Omega Y} \phi w \partial_{t} s_{\alpha} d x d y+\int_{\Omega} \int_{Y} \phi \beta_{\alpha} s_{\alpha} w \partial_{t} p_{\alpha} d x d y=-\frac{1}{\varepsilon^{2}} \int_{\Omega} \int_{Y} K \lambda_{\alpha}\left(\partial_{y i} p_{\alpha}+\varepsilon \partial_{x i} p_{\alpha}\right)\left(\partial_{y i} w+\varepsilon \partial_{x i} w\right) d y d x= \\
& =-\frac{1}{\varepsilon^{2}} \int_{\Omega Y^{\mathcal{F}}} K^{\mathcal{F}} \lambda_{\alpha}^{\mathcal{F}}\left(\partial_{y i} p_{\alpha}+\varepsilon \partial_{x i} p_{\alpha}\right)\left(\partial_{y i} w+\varepsilon \partial_{x i} w\right) d y d x- \\
& \quad-\frac{1}{\sqrt{\varepsilon}} \int_{\Omega Y^{\mathcal{M}}} \int^{\mathcal{M}} \lambda_{\alpha}^{\mathcal{M}}\left(\partial_{y i} p_{\alpha}+\varepsilon \partial_{x i} p_{\alpha}\right)\left(\partial_{y i} w+\varepsilon \partial_{x i} w\right) d y d x
\end{aligned}
$$

for any function $w=w(x, y)$ in $\Omega \times Y$, such that $\left.w\right|_{\partial \Omega}=0, w$ is continuous and periodic in $y$.

Relationship (2.5) is obtained by multiplying (2.3) by $w$, integrating by parts, and using the Gauss-Ostrogradsky theorem. The integrals arising in this case along the boundary of the domain $\partial \Omega$ are zeroed due to the fact that the function $w$ is equal to zero on $\partial \Omega$. The integrals over the period boundary $\partial Y$ are zeroed out due to the periodicity of all functions with respect to $y$.

2.3 Asymptotic expansion. Homogenization is performed by the method of two-scale asymptotic expansions over parameter $\varepsilon$, as well as regular asymptotic series over the nonlocality parameter $\omega$, which, taking into account (2.2), means the occurrence of terms containing fractional powers $\sqrt{\varepsilon}$. The general structure of the expansion is as follows, for $\alpha=w, o$ :

$$
\begin{gathered}
p_{\alpha}(x, y, t)= \begin{cases}p_{\alpha 0}(x, t)+\sqrt{\varepsilon} p_{\alpha, 1 / 2}^{\mathcal{M}}(x, y, t)+\varepsilon p_{\alpha 1}^{\mathcal{M}}(x, y, t)+\ldots, & y \in Y^{\mathcal{M}} \\
p_{\alpha 0}(x, t)+\sqrt{\varepsilon} p_{\alpha, 1 / 2}^{\mathcal{F}}(x, t)+\varepsilon p_{\alpha 1}^{\mathcal{F}}(x, y, t)+\ldots, & y \in Y^{\mathcal{F}}\end{cases} \\
s_{\alpha}(x, y, t)= \begin{cases}s_{\alpha 0}(x, t)+\sqrt{\varepsilon} s_{\alpha, 1 / 2}^{\mathcal{M}}(x, y, t)+\varepsilon s_{\alpha 1}^{\mathcal{M}}(x, y, t)+\ldots, & y \in Y^{\mathcal{M}} \\
s_{\alpha 0}(x, t)+\sqrt{\varepsilon} s_{\alpha, 1 / 2}^{\mathcal{F}}(x, t)+\varepsilon s_{\alpha 1}^{\mathcal{F}}(x, y, t)+\ldots, & y \in Y^{\mathcal{F}}\end{cases}
\end{gathered}
$$


The phase pressures should be continuous:

$$
\left.p_{\alpha, 1 / 2}^{\mathcal{M}}\right|_{y \in \Gamma}=p_{\alpha, 1 / 2}^{\mathcal{F}}(x, t)
$$

(The independence of the first terms of $y$ is easily proved by substituting the expansion in (2.5)).

Then, for the nonlinear functions in (2.5), we obtain:

$$
\lambda_{\alpha}(x, y, t)=\left\{\begin{array}{l}
\lambda_{\alpha 0}(x, t)+\sqrt{\varepsilon} \lambda_{\alpha, 1 / 2}^{\mathcal{M}}(x, y, t)+\varepsilon \ldots, \quad y \in Y^{\mathcal{M}} \\
\lambda_{\alpha 0}(x, t)+\sqrt{\varepsilon} \lambda_{\alpha, 1 / 2}^{\mathcal{F}}(x, t)+\varepsilon \ldots, \quad y \in Y^{\mathcal{F}}
\end{array}\right.
$$

Integral identity (2.5) takes the form:

$$
\begin{aligned}
\iint_{\Omega Y} \phi w & \left(\partial_{t} s_{\alpha 0}+\sqrt{\varepsilon} \partial_{t} s_{\alpha 1 / 2}+\ldots\right) d x d y+\iint_{\Omega Y} \phi \beta_{\alpha} w\left(s_{\alpha 0}+\sqrt{\varepsilon} s_{\alpha 1 / 2}+\ldots\right)\left(\partial_{t} p_{\alpha 0}+\sqrt{\varepsilon} \partial_{t} p_{\alpha 1 / 2}+\ldots\right) d x d y= \\
= & -\frac{1}{\varepsilon} \int_{\Omega Y^{\mathcal{F}}} K^{\mathcal{F}}\left(\lambda_{\alpha 0}^{\mathcal{F}}+\sqrt{\varepsilon} \lambda_{\alpha 1 / 2}^{\mathcal{F}}+\ldots\right)\left(\partial_{y i} p_{\alpha 1}+\partial_{x i} p_{\alpha 0}+\sqrt{\varepsilon}\left(\partial_{y i} p_{\alpha 3 / 2}+\partial_{x i} p_{\alpha 1 / 2}\right)\right)\left(\partial_{y i} w+\varepsilon \partial_{x i} w\right) d y d x- \\
& -\int_{\Omega Y^{\mathcal{M}}} \int^{\mathcal{M}} \lambda_{\alpha 0}^{\mathcal{M}}\left(\partial_{y i} p_{\alpha 1 / 2}+\sqrt{\varepsilon}\left(\partial_{y i} p_{\alpha 1}+\partial_{x i} p_{\alpha 0}\right)\right)\left(\partial_{y i} w+\varepsilon \partial_{x i} w\right) d y d x+\mathcal{O}(\varepsilon)
\end{aligned}
$$

The general technique of homogenization consists in substituting the asymptotic expansion into the integral identity (2.8) and obtaining closed expressions for successive terms of the expansion by choosing test functions $w$.

2.4 The result of homogenization - the macroscopic model. We immediately give the result of homogenization. Its deduction is given in the next section.

We define the averaged phase pressures and saturations in blocks and fractures as: 


$$
\begin{aligned}
& \mathcal{P}_{\alpha}^{\mathcal{F}} \equiv \frac{1}{\left|Y^{\mathcal{F}}\right|_{Y^{\mathcal{F}}}}\left(p_{\alpha 0}+\sqrt{\varepsilon} p_{\alpha 1 / 2}^{\mathcal{F}}\right) d y=p_{\alpha 0}+\sqrt{\varepsilon} p_{\alpha 1 / 2}^{\mathcal{F}}, \\
& \mathcal{P}_{\alpha}^{\mathcal{M}} \equiv \frac{1}{\left|Y^{\mathcal{M}}\right|} \int_{Y^{\mathcal{M}}}\left(p_{\alpha 0}+\sqrt{\varepsilon} p_{\alpha 1 / 2}^{\mathcal{M}}\right) d y=p_{\alpha 0}+\sqrt{\varepsilon}\left\langle p_{\alpha 1 / 2}^{\mathcal{M}}\right\rangle_{\mathcal{M}} \\
& \mathcal{S}_{\alpha}^{\mathcal{F}} \equiv \frac{1}{\left|Y^{\mathcal{F}}\right|} \int_{Y^{\mathcal{F}}}\left(s_{\alpha 0}+\sqrt{\varepsilon} s_{\alpha 1 / 2}^{\mathcal{F}}\right) d y=s_{\alpha 0}+\sqrt{\varepsilon} s_{\alpha 1 / 2}^{\mathcal{F}}, \\
& \mathcal{S}_{\alpha}^{\mathcal{M}} \equiv \frac{1}{\left|Y^{\mathcal{M}}\right|_{Y^{\mathcal{M}}}}\left(s_{\alpha 0}+\sqrt{\varepsilon} s_{\alpha 1 / 2}^{\mathcal{M}}\right) d y=s_{\alpha 0}+\sqrt{\varepsilon}\left\langle s_{\alpha 1 / 2}^{\mathcal{M}}\right\rangle_{\mathcal{M}}
\end{aligned}
$$

where

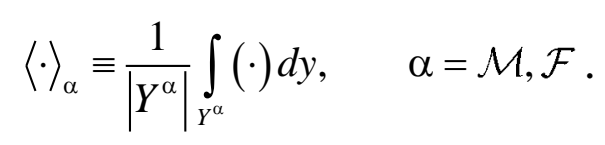

The following links exist between them:

$$
\mathcal{S}_{o}^{\mathcal{F}}=1-\mathcal{S}_{w}^{\mathcal{F}}, \quad \mathcal{S}_{o}^{\mathcal{M}}=1-\mathcal{S}_{w}^{\mathcal{M}}, \quad \mathcal{P}_{o}^{\mathcal{F}}=\mathcal{P}_{w}^{\mathcal{F}}+p_{c}^{\mathcal{F}}\left(\mathcal{S}_{w}^{\mathcal{F}}\right), \quad \mathcal{P}_{o}^{\mathcal{M}}=\mathcal{P}_{w}^{\mathcal{M}}+p_{c}^{\mathcal{M}}\left(\mathcal{S}_{w}^{\mathcal{M}}\right)
$$

Note that macroscopic capillary pressures in fractures and blocks, $\mathcal{P}_{c}^{\mathcal{F}}\left(\mathcal{S}_{w}^{\mathcal{F}}\right)$ and $\mathcal{P}_{c}^{\mathcal{M}}\left(\mathcal{S}_{w}^{\mathcal{M}}\right)$, do not arise in this model, since they are simply equal to the original capillary pressure as the function of average saturations, with accuracy $\mathcal{O}(\varepsilon)$ :

$$
\mathcal{P}_{c}^{\mathcal{F}}\left(\mathcal{S}_{w}^{\mathcal{F}}\right)=p_{c}^{\mathcal{F}}\left(\mathcal{S}_{w}^{\mathcal{F}}\right), \quad \mathcal{P}_{c}^{\mathcal{M}}\left(\mathcal{S}_{w}^{\mathcal{M}}\right)=p_{c}^{\mathcal{M}}\left(\mathcal{S}_{w}^{\mathcal{M}}\right)
$$

The macroscopic model of the process has the form of the following system of equations for average pressures and saturations in blocks and fractures:

$$
\begin{gathered}
\phi^{\mathcal{F}}(1-\theta) \partial_{t} \mathcal{S}_{\alpha}^{\mathcal{F}}+\phi^{\mathcal{F}} \beta_{\alpha}^{\mathcal{F}}(1-\theta) \mathcal{S}_{\alpha}^{\mathcal{F}} \partial_{t} \mathcal{P}_{\alpha}^{\mathcal{F}}= \\
=\partial_{x i}\left(\mathbb{K}_{i k} \lambda_{\alpha}^{\mathcal{F}}\left(\mathcal{S}_{\alpha}^{\mathcal{F}}\right) \partial_{x k} \mathcal{P}_{\alpha}^{\mathcal{F}}\right)+\xi_{\alpha}\left(\mathcal{P}_{\alpha}^{\mathcal{M}}-\mathcal{P}_{\alpha}^{\mathcal{F}}\right), \quad \alpha=w, o \\
\mathcal{P}_{w}^{\mathcal{M}}=\mathcal{P}_{w}^{\mathcal{F}}-\frac{\tau_{w}^{\text {comp }}}{\beta_{w}^{\mathcal{M}} \mathcal{S}_{w}^{\mathcal{M}}}\left[\partial_{t} \mathcal{S}_{w}^{\mathcal{M}}+\beta_{w}^{\mathcal{M}} \mathcal{S}_{w}^{\mathcal{M}} \partial_{t} \mathcal{P}_{w}^{\mathcal{M}}\right] \\
p_{c}^{\mathcal{M}}\left(\mathcal{S}_{w}^{\mathcal{M}}\right)=p_{c}^{\mathcal{F}}\left(\mathcal{S}_{w}^{\mathcal{F}}\right)+\frac{\left(\tau^{\mathrm{cap}}+\tau^{\mathrm{cc}}\right)}{\beta_{o}^{\mathcal{M}}} \partial_{t} \mathcal{S}_{w}^{\mathcal{M}}+\left(\tau_{w}^{\text {comp }}-\tau_{o}^{\text {comp }}\right) \partial_{t} \mathcal{P}_{w}^{\mathcal{M}}
\end{gathered}
$$


where $\theta \equiv\left|Y^{\mathcal{M}}\right|$ is the volume fraction of blocks.

The effective permeability is defined as:

$$
\mathbb{K}_{i k} \equiv \int_{Y^{\mathcal{F}}} K^{\mathcal{F}}\left(\frac{\partial \psi_{k}}{\partial y_{i}}+\delta_{i k}\right) d y
$$

and the cell function $\psi_{k}(y)$ is the solution to the first cell problem (in the fracture), for $k=1,2,3:$

$$
\left\{\begin{array}{l}
\frac{\partial}{\partial y_{i}}\left(K^{\mathcal{F}}\left(\frac{\partial \psi_{k}}{\partial y_{i}}+\delta_{i k}\right)\right)=0, \quad y \in Y^{\mathcal{F}} \\
\left.K^{\mathcal{F}}\left(\frac{\partial \psi_{k}}{\partial y_{i}}+\delta_{i k}\right) n_{i}^{\Gamma}\right|_{y \in \Gamma}=0, \\
\psi_{k} \text { y-periodic } \\
\left\langle\psi_{k}\right\rangle_{Y^{\mathcal{F}}}=0
\end{array}\right.
$$

The delay times between blocks and fractures caused by phase compressibility are defined as:

$$
\tau_{\alpha}^{\text {comp }}\left(\mathcal{S}_{w}^{\mathcal{M}}\right)=\sqrt{\varepsilon}\langle\varphi\rangle_{\mathcal{M}} \phi^{\mathcal{M}} \frac{\beta_{\alpha}^{\mathcal{M}} \mathcal{S}_{\alpha}^{\mathcal{M}}}{\lambda_{\alpha}^{\mathcal{M}}}
$$

The delay times caused by capillarity and the combined action of capillarity and compressibility are defined as:

$$
\begin{aligned}
\tau^{\mathrm{cap}}\left(\mathcal{S}_{w}^{\mathcal{M}}\right) & =\sqrt{\varepsilon}\langle\varphi\rangle_{\mathcal{M}} \phi^{\mathcal{M}} \beta_{\mathrm{o}}^{\mathcal{M}} \frac{\left(\lambda_{w}^{\mathcal{M}}+\lambda_{o}^{\mathcal{M}}\right)}{\lambda_{w}^{\mathcal{M}} \lambda_{o}^{\mathcal{M}}} \\
\tau^{\mathrm{cc}}\left(\mathcal{S}_{w}^{\mathcal{M}}\right) & =-\beta_{\mathrm{o}}^{\mathcal{M}} \frac{d p_{c}^{\mathcal{M}}}{d \mathcal{S}_{w}^{\mathcal{M}}} \tau_{o}^{\text {comp }}
\end{aligned}
$$

The function $\varphi(y)$ is the solution of the second cell problem (in the block): 


$$
\left\{\begin{array}{l}
\frac{\partial}{\partial y_{i}}\left(K^{\mathcal{M}} \frac{\partial \varphi}{\partial y_{i}}\right)=-1, \quad y \in Y^{\mathcal{M}} \\
\left.\varphi\right|_{y \in \Gamma}=0
\end{array}\right.
$$

Parameters of mass exchange $\xi_{\alpha}$ are defined as follows:

$$
\xi_{\alpha}=\frac{\theta \lambda_{\alpha}^{\mathcal{M}}}{\sqrt{\varepsilon}\langle\varphi\rangle_{\mathcal{M}}}
$$

Model (2.10) is a formal asymptotic expansion of the original problem, preserving the terms of the order $\mathcal{O}(\sqrt{\varepsilon})$. The order of the cut-off terms is thus $\mathcal{O}(\varepsilon)$.

\section{Derivation of the averaged model}

3.1 First step: homogenization in the fracture. Let $w=w(x, y)$ in $\Omega \times Y,\left.w\right|_{\alpha \Omega}=0, w$ be continuous and periodic in $y$. Then we obtain from (2.8) for negative powers $\varepsilon$ :

$$
\begin{aligned}
& 0=\frac{1}{\varepsilon} \int_{\Omega Y^{\mathcal{F}}} \int^{\mathcal{F}} \lambda_{\alpha 0}\left(\partial_{y i} p_{\alpha 1}^{\mathcal{F}}+\partial_{x i} p_{\alpha 0}\right) \partial_{y i} w d y d x \\
& 0=\frac{1}{\sqrt{\varepsilon}} \int_{\Omega Y^{\mathcal{F}}} \int^{\mathcal{F}}\left[\lambda_{\alpha 0}\left(\partial_{y i} p_{\alpha 3 / 2}^{\mathcal{F}}+\partial_{x i} p_{\alpha 1 / 2}^{\mathcal{F}}\right)+\lambda_{\alpha 1 / 2}^{\mathcal{F}}\left(\partial_{y i} p_{\alpha 1}^{\mathcal{F}}+\partial_{x i} p_{\alpha 0}\right)\right] \partial_{y i} w d y d x
\end{aligned}
$$

It follows from (3.1):

$$
\begin{aligned}
& 0=-\int_{\Omega Y^{\mathcal{F}}} w \partial_{y i}\left(K^{\mathcal{F}} \lambda_{\alpha 0}\left(\partial_{y i} p_{\alpha 1}^{\mathcal{F}}+\partial_{x i} p_{\alpha 0}\right)\right) d x d y+\iint_{\Omega \Gamma} w K^{\mathcal{F}} \lambda_{\alpha 0}\left(\partial_{y i} p_{\alpha 1}^{\mathcal{F}}+\partial_{x i} p_{\alpha 0}\right) n_{i}^{\Gamma} d x d y, \\
& 0=\int_{\Omega Y^{\mathcal{F}}} w \partial_{y i}\left(K^{\mathcal{F}} \lambda_{\alpha 0}\left(\partial_{y i} p_{\alpha 3 / 2}^{\mathcal{F}}+\partial_{x i} p_{\alpha 1 / 2}^{\mathcal{F}}\right)+K^{\mathcal{F}} \lambda_{\alpha 1}^{\mathcal{F}}\left(\partial_{y i} p_{\alpha 1}^{\mathcal{F}}+\partial_{x i} p_{\alpha 0}\right)\right) d y d x+ \\
& +\iint_{\Omega \Gamma} w\left(K^{\mathcal{F}} \lambda_{\alpha 0}\left(\partial_{y i} p_{\alpha 3 / 2}^{\mathcal{F}}+\partial_{x i} p_{\alpha 1 / 2}^{\mathcal{F}}\right)+K^{\mathcal{F}} \lambda_{\alpha 1}^{\mathcal{F}}\left(\partial_{y i} p_{\alpha 1}^{\mathcal{F}}+\partial_{x i} p_{\alpha 0}\right)\right) n_{i}^{\Gamma} d x d y
\end{aligned}
$$

where $\mathbf{n}^{\Gamma}=\left\{n_{1}^{\Gamma}, n_{2}^{\Gamma}, n_{3}^{\Gamma}\right\}$ is is the unit normal vector to the boundary $\Gamma$ directed from the fracture to the block. 
In view of (2.7) and the fact that $\lambda_{\alpha 0}$ does not depend on $y$, this is equivalent to two problems in classical formulation:

$$
\left\{\begin{array}{l}
\partial_{y i}\left(K^{\mathcal{F}}\left(\partial_{y i} p_{\alpha 1}^{\mathcal{F}}+\partial_{x i} p_{\alpha 0}\right)\right)=0, \quad y \in Y^{\mathcal{F}} \\
\left.K^{\mathcal{F}}\left(\partial_{y i} p_{\alpha 1}^{\mathcal{F}}+\partial_{x i} p_{\alpha 0}\right) n_{i}^{\Gamma}\right|_{y \in \Gamma}=0
\end{array}, \quad\left\{\begin{array}{l}
\partial_{y i}\left(K^{\mathcal{F}}\left(\partial_{y i} p_{\alpha 3 / 2}^{\mathcal{F}}+\partial_{x i} p_{\alpha 1 / 2}^{\mathcal{F}}\right)\right)=0, \quad y \in Y^{\mathcal{F}} \\
\left.K^{\mathcal{F}}\left(\partial_{y i} p_{\alpha 3 / 2}^{\mathcal{F}}+\partial_{x i} p_{\alpha 1 / 2}^{\mathcal{F}}\right) n_{i}^{\Gamma}\right|_{y \in \Gamma}=0
\end{array}\right.\right.
$$

This gives the following representation for $p_{\alpha 1}^{\mathcal{F}}$ and $p_{\alpha 3 / 2}^{\mathcal{F}}$ through $p_{\alpha 0}$ and $p_{\alpha 1 / 2}^{\mathcal{F}}$ :

$$
p_{\alpha 1}^{\mathcal{F}}=\psi_{k}(y) \frac{\partial p_{\alpha 0}}{\partial x_{k}}+\bar{p}_{\alpha 1}^{\mathcal{F}}(x, t), \quad \quad p_{\alpha 3 / 2}^{\mathcal{F}}=\psi_{k}(y) \frac{\partial p_{\alpha 1 / 2}^{\mathcal{F}}}{\partial x_{k}}+\bar{p}_{\alpha 3 / 2}^{\mathcal{F}}(x, t)
$$

where $\bar{p}_{\alpha 1}^{\mathcal{F}}(x, t)$ and $\bar{p}_{\alpha 3 / 2}^{\mathcal{F}}(x, t)$ are some slow functions, which will not be included in the averaged model. For functions $\psi_{k}(y)$, we obtain the first cell problem (2.12). The last condition in it is added for the uniqueness of the solution.

3.2 Second step - averaged equation in fractures. Let $w=w(x)$ in $\Omega$ and $\left.w\right|_{\partial \Omega}=0$.

Then (2.8) gives:

$$
\begin{aligned}
& \iint_{\Omega Y} w \phi\left(\partial_{t} s_{\alpha 0}+\sqrt{\varepsilon} \partial_{t} s_{\alpha 1 / 2}\right) d x d y+\iint_{\Omega Y} w \phi \beta_{\alpha}\left(s_{\alpha 0}+\sqrt{\varepsilon} s_{\alpha 1 / 2}\right)\left(\partial_{t} p_{\alpha 0}+\sqrt{\varepsilon} \partial_{t} p_{\alpha 1 / 2}\right) d x d y= \\
& \quad=-\int_{\Omega Y^{\mathcal{F}}} K^{\mathcal{F}}\left(\lambda_{\alpha 0}^{\mathcal{F}}+\sqrt{\varepsilon} \lambda_{\alpha 1 / 2}^{\mathcal{F}}\right)\left(\partial_{y i} p_{\alpha 1}+\partial_{x i} p_{\alpha 0}+\sqrt{\varepsilon}\left(\partial_{y i} p_{\alpha 3 / 2}+\partial_{x i} p_{\alpha 1 / 2}\right)\right) \partial_{x i} w d y d x+\mathcal{O}(\varepsilon)
\end{aligned}
$$

Or, taking into account (3.3):

$$
\begin{array}{r}
\iint_{\Omega Y} w \phi \partial_{t}\left(s_{\alpha 0}+\sqrt{\varepsilon} s_{\alpha 1 / 2}\right) d x d y+\iint_{\Omega Y} w \phi \beta_{\alpha}\left(s_{\alpha 0}+\sqrt{\varepsilon} s_{\alpha 1 / 2}\right) \partial_{t}\left(p_{\alpha 0}+\sqrt{\varepsilon} p_{\alpha 1 / 2}\right) d x d y= \\
\quad=\int_{\Omega Y^{\mathcal{F}}} w \partial_{x i}\left(K^{\mathcal{F}}\left(\lambda_{\alpha 0}+\sqrt{\varepsilon} \lambda_{\alpha 1 / 2}^{\mathcal{F}}\right)\left(\partial_{y i} \psi_{k}+\delta_{i k}\right) \partial_{x k}\left(p_{\alpha 0}+\sqrt{\varepsilon} p_{\alpha 1 / 2}^{\mathcal{F}}\right)\right) d y d x+\mathcal{O}(\varepsilon)
\end{array}
$$

Introducing the average pressure and saturation (2.9), we obtain: 


$$
\begin{gathered}
\int_{\Omega} w\left[\phi^{\mathcal{F}}(1-\theta) \partial_{t} \mathcal{S}_{\alpha}^{\mathcal{F}}+\phi^{\mathcal{M}} \theta \partial_{t} \mathcal{S}_{\alpha}^{\mathcal{M}}\right] d x+\int_{\Omega} w\left[\phi^{\mathcal{F}} \beta_{\alpha}^{\mathcal{F}}(1-\theta) \mathcal{S}_{\alpha}^{\mathcal{F}} \partial_{t} \mathcal{P}_{\alpha}^{\mathcal{F}}+\phi^{\mathcal{M}} \beta_{\alpha}^{\mathcal{M}} \theta \mathcal{S}_{\alpha}^{\mathcal{M}} \partial_{t} \mathcal{P}_{\alpha}^{\mathcal{M}}\right] d x= \\
=\int_{\Omega} w \partial_{x i}\left(\mathbb{K}_{i k} \lambda_{\alpha}^{\mathcal{F}}\left(\mathcal{S}_{\alpha}^{\mathcal{F}}\right) \partial_{x k} \mathcal{P}_{\alpha}^{\mathcal{F}}\right) d x, \quad \alpha=w, o
\end{gathered}
$$

where the effective permeability is (2.11). This gives the first averaged equation of the system (2.9)-a in almost complete form:

$$
\phi^{\mathcal{F}}(1-\theta) \partial_{t} \mathcal{S}_{\alpha}^{\mathcal{F}}+\phi^{\mathcal{F}} \beta_{\alpha}^{\mathcal{F}}(1-\theta) \mathcal{S}_{\alpha}^{\mathcal{F}} \partial_{t} \mathcal{P}_{\alpha}^{\mathcal{F}}=\partial_{x i}\left(\mathbb{K}_{i k} \lambda_{\alpha}^{\mathcal{F}}\left(\mathcal{S}_{\alpha}^{\mathcal{F}}\right) \partial_{x k} \mathcal{P}_{\alpha}^{\mathcal{F}}\right)-\phi^{\mathcal{M}} \theta \partial_{t} \mathcal{S}_{\alpha}^{\mathcal{M}}-\phi^{\mathcal{M}} \beta_{\alpha}^{\mathcal{M}} \theta \mathcal{S}_{\alpha}^{\mathcal{M}} \partial_{t} \mathcal{P}_{\alpha}^{\mathcal{M}}
$$

3.3 Third step - expansion in the blocks. Let $w=w(x, y)$ in $\Omega \times Y,\left.w\right|_{\partial \Omega}=0$, $w \equiv 0$ in $Y^{\mathcal{F}}$, and $w$ is continuous function of both arguments. Then (2.8) gives for terms of the zeroth order:

$$
\begin{gathered}
\iint_{\Omega Y^{\mathcal{M}}} w \phi\left(\partial_{t} s_{\alpha 0}+\sqrt{\varepsilon} \partial_{t} s_{\alpha 1 / 2}^{\mathcal{M}}\right) d x d y+\int_{\Omega} \int_{Y^{\mathcal{M}}} w \phi \beta_{\alpha}\left(s_{\alpha 0}+\sqrt{\varepsilon} s_{\alpha 1 / 2}^{\mathcal{M}}\right)\left(\partial_{t} p_{\alpha 0}+\sqrt{\varepsilon} \partial_{t} p_{\alpha 1 / 2}^{\mathcal{M}}\right) d x d y= \\
=-\int_{\Omega} \int_{Y^{\mathcal{M}}} K^{\mathcal{M}}\left(\lambda_{\alpha 0}+\sqrt{\varepsilon} \lambda_{\alpha 1 / 2}^{\mathcal{M}}\right)\left(\partial_{y i} p_{\alpha 1 / 2}^{\mathcal{M}}+\sqrt{\varepsilon}\left(\partial_{y i} p_{\alpha 1 / 2}^{\mathcal{M}}+\partial_{x i} p_{\alpha 0}\right)\right) \partial_{y i} w d y d x+\mathcal{O}(\varepsilon)
\end{gathered}
$$

The integral along the block boundary $\Gamma$ is equal to zero, since the functions $w$ are zeroth in the fractures and are continuous.

Zero-order terms give:

$$
\int_{\Omega} \int_{Y^{\mathcal{M}}} w \phi\left(\partial_{t} s_{\alpha 0}+\beta_{\alpha} s_{\alpha 0} \partial_{t} p_{\alpha 0}\right) d y d x=\int_{\Omega} \int_{Y^{\mathcal{M}}} w \partial_{y i}\left(K^{\mathcal{M}} \lambda_{\alpha 0} \partial_{y i} p_{\alpha 1 / 2}^{\mathcal{M}}\right) d y d x
$$

which defines the following expression for $p_{\alpha 1 / 2}^{\mathcal{M}}$ taking into account (2.6):

$$
p_{\alpha 1 / 2}^{\mathcal{M}}=p_{\alpha 1 / 2}^{\mathcal{F}}(x, t)-\varphi(y) \frac{\phi^{\mathcal{M}}}{\lambda_{\alpha 0}}\left[\partial_{t} s_{\alpha 0}+\beta_{\alpha}^{\mathcal{M}} s_{\alpha 0} \partial_{t} p_{\alpha 0}\right]
$$


where $\varphi(y)$ is the solution to the second cell problem (2.14). The boundary condition in (2.14) results from the condition of continuity of phase pressures (2.6).

3.4 Fourth step - averaged equation in the blocks. Relationship (3.4) makes it possible to obtain an explicit relationship between $\mathcal{P}_{\alpha}^{\mathcal{M}}$ and $\mathcal{P}_{\alpha}^{\mathcal{F}}$. In fact, taking the average of (3.4) over $Y^{\mathcal{M}}$ (and taking into account that on the right-hand side of (3.4) only the function $\varphi(y)$ depends on $y$ ), multiplying by $\sqrt{\varepsilon}$ and adding $p_{\alpha 0}$, we get:

$$
p_{\alpha 0}+\sqrt{\varepsilon}\left\langle p_{\alpha 1 / 2}^{\mathcal{M}}\right\rangle_{\mathcal{M}}=p_{\alpha 0}+\sqrt{\varepsilon} p_{\alpha 1 / 2}^{\mathcal{F}}-\sqrt{\varepsilon}\langle\varphi\rangle_{\mathcal{M}} \frac{\phi^{\mathcal{M}}}{\lambda_{\alpha 0}}\left[\partial_{t} s_{\alpha 0}+\beta_{\alpha}^{\mathcal{M}} s_{\alpha 0} \partial_{t} p_{\alpha 0}\right]
$$

Given the definition of average pressures and saturations (2.9), we obtain:

$$
\mathcal{P}_{\alpha}^{\mathcal{M}}=\mathcal{P}_{\alpha}^{\mathcal{F}}-\frac{\tau_{\alpha}^{\text {comp }}}{\beta_{\alpha}^{\mathcal{M}} \mathcal{S}_{\alpha}^{\mathcal{M}}}\left[\partial_{t} \mathcal{S}_{\alpha}^{\mathcal{M}}+\beta_{\alpha}^{\mathcal{M}} \mathcal{S}_{\alpha}^{\mathcal{M}} \partial_{t} \mathcal{P}_{\alpha}^{\mathcal{M}}\right], \quad \alpha=w, o
$$

which corresponds to (2.10)-b. The characteristic delay times are defined as (2.13) -a.

In the structure of delay times, we took into account the fact that

$$
\begin{gathered}
\partial_{t} s_{\alpha 0}=\partial_{t}\left(s_{\alpha 0}+\sqrt{\varepsilon}\left\langle s_{\alpha 1 / 2}^{\mathcal{M}}\right\rangle_{\mathcal{M}}\right)-\sqrt{\varepsilon} \partial_{t}\left\langle s_{\alpha 1 / 2}^{\mathcal{M}}\right\rangle_{\mathcal{M}}=\partial_{t} \mathcal{S}_{\alpha}^{\mathcal{M}}+\mathcal{O}(\sqrt{\varepsilon}), \\
\lambda_{\alpha 0}^{\mathcal{M}} \equiv \lambda_{\alpha}^{\mathcal{M}}\left(s_{\alpha 0}\right)=\lambda_{\alpha}^{\mathcal{M}}\left(s_{\alpha 0}\right)+\sqrt{\varepsilon} \frac{d \lambda_{\alpha}^{\mathcal{M}}}{d s_{\alpha 0}}\left\langle s_{\alpha 1 / 2}^{\mathcal{M}}\right\rangle_{\mathcal{M}}-\sqrt{\varepsilon} \frac{d \lambda_{\alpha}^{\mathcal{M}}}{d s_{\alpha 0}}\left\langle s_{\alpha 1 / 2}^{\mathcal{M}}\right\rangle_{\mathcal{M}}= \\
=\lambda_{\alpha}^{\mathcal{M}}\left(s_{\alpha 0}+\sqrt{\varepsilon}\left\langle s_{\alpha 1 / 2}^{\mathcal{M}}\right\rangle_{\mathcal{M}}\right)+\mathcal{O}(\sqrt{\varepsilon})=\lambda_{\alpha}^{\mathcal{M}}\left(\mathcal{S}_{\alpha}^{\mathcal{M}}\right)+\mathcal{O}(\sqrt{\varepsilon})
\end{gathered}
$$

As a result, we get: 


$$
\frac{\sqrt{\varepsilon}}{\lambda_{\alpha 0}^{\mathcal{M}}} \partial_{t} s_{\alpha 0}=\frac{\sqrt{\varepsilon} \partial_{t} \mathcal{S}_{\alpha}^{\mathcal{M}}+\mathcal{O}(\varepsilon)}{\lambda_{\alpha}^{\mathcal{M}}\left(\mathcal{S}_{\alpha}^{\mathcal{M}}\right)+\mathcal{O}(\sqrt{\varepsilon})}=\frac{\sqrt{\varepsilon} \partial_{t} \mathcal{S}_{\alpha}^{\mathcal{M}}}{\lambda_{\alpha}^{\mathcal{M}}\left(\mathcal{S}_{\alpha}^{\mathcal{M}}\right)}+\mathcal{O}(\varepsilon)
$$

etc. for other terms.

Instead of the two equations (3.5) for phase pressures, it is more convenient to replace one of them with the equation for capillary pressure. Subtracting one equation (3.5) from the other, we obtain the equation of coupling between the average capillary pressures in blocks and fractures:

$$
\begin{aligned}
\mathcal{P}_{c}^{\mathcal{M}}= & \mathcal{P}_{c}^{\mathcal{F}}+\tau_{w}^{\text {comp }} \partial_{t} \mathcal{P}_{w}^{\mathcal{M}}-\tau_{o}^{\text {comp }} \partial_{t}\left(\mathcal{P}_{w}^{\mathcal{M}}+\mathcal{P}_{c}^{\mathcal{M}}\right)+\left(\frac{\tau_{w}^{\text {comp }}}{\beta_{w}^{\mathcal{M}} \mathcal{S}_{w}^{\mathcal{M}}}+\frac{\tau_{o}^{\text {comp }}}{\beta_{o}^{\mathcal{M}} \mathcal{S}_{o}^{\mathcal{M}}}\right) \partial_{t} \mathcal{S}_{w}^{\mathcal{M}}= \\
& =\mathcal{P}_{c}^{\mathcal{F}}+\left(\frac{\tau_{w}^{\text {comp }}}{\beta_{w}^{\mathcal{M}} \mathcal{S}_{w}^{\mathcal{M}}}+\frac{\tau_{o}^{\text {comp }}}{\beta_{o}^{\mathcal{M}} \mathcal{S}_{o}^{\mathcal{M}}}-\tau_{o}^{\text {comp }} \frac{d \mathcal{P}_{c}^{\mathcal{M}}}{d \mathcal{S}_{w}^{\mathcal{M}}}\right) \frac{\partial \mathcal{S}_{w}^{\mathcal{M}}}{\partial t}+\left(\tau_{w}^{\text {comp }}-\tau_{o}^{\text {comp p }}\right) \frac{\partial \mathcal{P}_{w}^{\mathcal{M}}}{\partial t}
\end{aligned}
$$

which is reduced to (2.10)-c if we associate the average capillary pressures $\mathcal{P}_{c}^{\mathcal{F}}$ and $\mathcal{P}_{c}^{\mathcal{M}}$ with the originally given capillary pressure curves $p_{c}^{\mathcal{F}}(s)$ and $p_{c}^{\mathcal{M}}(s)$, that is, we obtain (2.9)-d. This is done in the following way. We expand the expression for $p_{c}^{\mathcal{F}}\left(\mathcal{S}_{w}^{\mathcal{F}}\right)$ and $p_{c}^{\mathcal{M}}\left(\mathcal{S}_{w}^{\mathcal{M}}\right)$ in the Taylor series, taking into account (2.9) -b:

$$
\begin{gathered}
p_{c}^{\mathcal{F}}\left(\mathcal{S}_{w}^{\mathcal{F}}\right)=p_{c}^{\mathcal{F}}\left(s_{w 0}\right)+\sqrt{\varepsilon} \frac{d p_{\alpha}^{\mathcal{F}}}{d s_{w 0}} s_{w 1 / 2}^{\mathcal{F}}+\mathcal{O}(\varepsilon) \\
p_{c}^{\mathcal{M}}\left(\mathcal{S}_{w}^{\mathcal{M}}\right)=p_{c}^{\mathcal{M}}\left(s_{w 0}\right)+\sqrt{\varepsilon} \frac{d p_{\alpha}^{\mathcal{M}}}{d s_{w 0}}\left\langle s_{w 1 / 2}^{\mathcal{M}}\right\rangle_{\mathcal{M}}+\mathcal{O}(\varepsilon)
\end{gathered}
$$

Since all functions on the right are independent of $y$, the average over the fracture and over the block does not change anything from these formulas, that is: 


$$
\mathcal{P}_{c}^{\mathcal{F}}\left(\mathcal{S}_{w}^{\mathcal{F}}\right) \equiv\left\langle p_{c}^{\mathcal{F}}\left(\mathcal{S}_{w}^{\mathcal{F}}\right)\right\rangle_{\mathcal{F}}=p_{c}^{\mathcal{F}}\left(\mathcal{S}_{w}^{\mathcal{F}}\right)+\mathcal{O}(\varepsilon), \quad \mathcal{P}_{c}^{\mathcal{M}}\left(\mathcal{S}_{w}^{\mathcal{M}}\right) \equiv\left\langle p_{c}^{\mathcal{M}}\left(\mathcal{S}_{w}^{\mathcal{M}}\right)\right\rangle_{\mathcal{M}}=p_{c}^{\mathcal{M}}\left(\mathcal{S}_{w}^{\mathcal{F}}\right)+\mathcal{O}(\varepsilon)
$$

which confirms (2.9)-d. This makes it possible to write formula (3.6) in terms of the originally given functions of capillary pressure: (2.10) -c.

For the expressions on the right-hand side of macroscopic equations (3.3), we can obtain an explicit expression through the pressure difference using (2.10)-b:

$$
\partial_{t} \mathcal{S}_{\alpha}^{\mathcal{M}}+\beta_{\alpha}^{\mathcal{M}} \mathcal{S}_{\alpha}^{\mathcal{M}} \partial_{t} \mathcal{P}_{\alpha}^{\mathcal{M}}=-\frac{\beta_{\alpha}^{\mathcal{M}} \mathcal{S}_{\alpha}^{\mathcal{M}}}{\tau_{\alpha}^{\text {comp }}}\left(\mathcal{P}_{\alpha}^{\mathcal{M}}-\mathcal{P}_{\alpha}^{\mathcal{F}}\right)
$$

which gives the final form of the averaged equations (2.10) -a.

\section{Physical analysis of the model}

Equations (2.10)-a describe the behaviour of the saturation $\mathcal{S}^{\mathcal{F}}$ and pressure $\mathcal{P}^{\mathcal{F}}$ in fractures with exchange terms on the right proportional to the difference of phase pressures. Relationships (2.10)-b,c are a system of two ordinary differential equations for saturation $\mathcal{S}^{\mathcal{M}}$ and pressure $\mathcal{P}^{\mathcal{M}}$ in blocks (with known saturation and pressure in fractures).

4.1 Special case of a single-phase compressible system. In the single-phase case, system (2.10) takes the form:

$$
\begin{aligned}
& \phi^{\mathcal{F}} \beta^{\mathcal{F}}(1-\theta) \partial_{t} \mathcal{P}^{\mathcal{F}}-\partial_{x i}\left(\mathbb{K}_{i k} \partial_{x k} \mathcal{P}^{\mathcal{F}}\right)=\xi\left(\mathcal{P}^{\mathcal{M}}-\mathcal{P}^{\mathcal{F}}\right) \\
& \mathcal{P}^{\mathcal{M}}-\mathcal{P}^{\mathcal{F}}=-\tau^{\text {comp }} \frac{\partial \mathcal{P}^{\mathcal{M}}}{\partial t}
\end{aligned}
$$


where $\xi \equiv \frac{\theta}{\sqrt{\varepsilon}\langle\varphi\rangle_{\mathcal{M}}}, \quad \tau^{\text {comp }}=\sqrt{\varepsilon}\langle\varphi\rangle_{\mathcal{M}} \phi^{\mathcal{M}} \beta^{\mathcal{M}} \mu, \quad \mu$ is the dynamic viscosity of liquid.

This is a well-known double porosity model obtained, for example, in [7, 20].

4.2 Special case of an incompressible two-phase system. In the incompressible case, system (2.10) takes the form:

$$
\begin{gathered}
\phi^{\mathcal{F}}(1-\theta) \partial_{t} \mathcal{S}_{\alpha}^{\mathcal{F}}-\partial_{x i}\left(\mathbb{K}_{i k} \lambda_{\alpha}^{\mathcal{F}}\left(\mathcal{S}_{\alpha}^{\mathcal{F}}\right) \partial_{x k} \mathcal{P}_{\alpha}^{\mathcal{F}}\right)=\xi_{\alpha}\left(\mathcal{P}_{\alpha}^{\mathcal{M}}-\mathcal{P}_{\alpha}^{\mathcal{F}}\right), \quad \alpha=w, o \\
\mathcal{P}_{w}^{\mathcal{M}}=\mathcal{P}_{w}^{\mathcal{F}}-\frac{\tau_{w}^{\text {comp }}}{\beta_{w}^{\mathcal{M}} \mathcal{S}_{w}^{\mathcal{M}}} \partial_{t} \mathcal{S}_{w}^{\mathcal{M}}, \\
p_{c}^{\mathcal{M}}\left(\mathcal{S}_{w}^{\mathcal{M}}\right)=p_{c}^{\mathcal{F}}+\frac{\tau^{\text {cap }}}{\beta_{\mathrm{o}}^{\mathcal{M}}} \partial_{t} \mathcal{S}_{w}^{\mathcal{M}}
\end{gathered}
$$

where the relationships $\frac{\tau_{w}^{\text {comp }}}{\beta_{w}^{\mathcal{M}} \mathcal{S}^{\mathcal{M}}}$ and $\frac{\tau^{\text {cap }}}{\beta_{\mathrm{o}}^{\mathcal{M}}}$ do not depend on the compressibility coefficients. In fact, according to (2.13)-a,b we obtain:

$$
\frac{\tau_{w}^{\text {comp }}}{\beta_{w}^{\mathcal{M}} \mathcal{S}^{\mathcal{M}}}=\sqrt{\varepsilon}\langle\varphi\rangle_{\mathcal{M}} \frac{\phi^{\mathcal{M}}}{\lambda_{w}^{\mathcal{M}}}, \quad \frac{\tau^{\mathrm{cap}}}{\beta_{o}^{\mathcal{M}}}=\sqrt{\varepsilon}\langle\varphi\rangle_{\mathcal{M}} \phi^{\mathcal{M}} \frac{\left(\lambda_{w}^{\mathcal{M}}+\lambda_{o}^{\mathcal{M}}\right)}{\lambda_{w}^{\mathcal{M}} \lambda_{o}^{\mathcal{M}}}
$$

As can be seen from two equations (4.2)-b,c, only the second is differential (with respect to the saturation in the blocks). The first one is algebraic with respect to the block pressure. This means that the delay in the system is caused only by capillarity, namely, by a strong difference in capillary effects in blocks and fractures. The difference in phase pressures also exists in this case, but only as a consequence of the link between phase pressures and capillary pressure.

This model coincides with that which was obtained in $[11,20]$ for an incompressible flow in a non-deformable medium. 
4.3 Capillary delay and delay caused by compressibility. Comparison with the case of incompressible fluids (4.2) and single-phase flow (4.1) allows better understanding of the essence of the obtained model (2.10) and the role of compressibility in a twophase system.

The nonequilibrium behavior of system (2.10) is determined by the subsystem of two ODEs (2.10)-b,c with respect to saturation $\mathcal{S}_{w}^{\mathcal{M}}$ and pressure $\mathcal{P}_{w}^{\mathcal{M}}$ in the blocks. The link of the solution in blocks and fractures through differential equations in time indicates the nonequilibrium nature of the process at the macroscopic scale. What happens at the microscale, what specific processes cause this disequilibrium? First of all, we distinguish two main mechanisms:

- Nonequilibrium capillary redistribution of the phases at the masroscale, caused by the fact that the average saturation in blocks changes more slowly than in fractures, which violates the equality of average capillary pressures (condition of capillary equilibrium). As a result, there is a difference in average capillary pressures, which depends on the rate of variation of saturation in the block. In equation (2.10)-c, this process is described by the first component: $p_{c}^{\mathcal{M}}-p_{c}^{\mathcal{F}}=\frac{\tau^{\text {cap }}}{\beta_{\mathrm{o}}^{\mathcal{M}}} \frac{\partial \mathcal{S}_{w}^{\mathcal{M}}}{\partial t}$. The difference in capillary pressures automatically causes the difference in phase pressures, which is described by the first term in (2.10)-b: $\mathcal{P}^{\mathcal{M}}-\mathcal{P}^{\mathcal{F}}=-\frac{\tau_{w}^{\text {comp }}}{\beta_{w}^{\mathcal{M}} \mathcal{S}_{w}^{\mathcal{M}}} \frac{\partial \mathcal{S}_{w}^{\mathcal{M}}}{\partial t}$. In a highly contrasting medium, such a process would lead to long-term memory. In the case of moderate contrast, which is under consideration, we obtain a short memory. This process is 
independent of the compressibility of phases and rocks and is the same as in the incompressible case (4.2)-b,c.

- Relaxation of pressure waves in compressible medium, caused by the fact that the pressure perturbation in low-permeable blocks propagates much more slowly than in fractures. This provokes a difference in average pressures, which is described by the second term in equation (2.10)-b: $\mathcal{P}^{\mathcal{M}}-\mathcal{P}^{\mathcal{F}}=-\tau_{w}^{\text {comp }} \frac{\partial \mathcal{P}^{\mathcal{M}}}{\partial t}$. This process is independent of the number of phases and is the same as in the single-phase case, (4.1).

Then cross effects arise from the imposition of compressibility and capillarity.

- Redistribution of phases due to asymmetric extrusion. Compressibility leads to the expansion of liquids and compaction of pores under the weight of overlying rocks (if the pressure drops), which leads to the "extrusion" of both phases from the pores. In geosciences, this effect is called "squeezing" the liquid from the pores. This effect is similar to peristalsis, when the liquid in the channel is squeezed out due to a sharp compression of the channel. If such extrusion occurs symmetrically for both phases, then they are squeezed out as a whole, which does not change their saturation. In the case of asymmetric extrusion, a phase redistribution starts, which affects the saturation. In blocks, this movement caused by extrusion is delayed, which introduces additional nonequilibrium in the behavior of saturation. An additional difference arises in capillary pressures, which is described by the third term in (2.10)-c: $p_{c}^{\mathcal{M}}-p_{c}^{\mathcal{F}}=\left(\tau_{w}^{\text {comp }}-\tau_{o}^{\text {comp }}\right) \frac{\partial \mathcal{P}_{w}^{\mathcal{M}}}{\partial t}$. As one can see, this effect is really zeroed with a symmetrical phase extraction, that is, when $\tau_{w}^{\text {comp }}=\tau_{o}^{\text {comp }}$. 


\section{- Redistribution of phases due to nonlinear extrusion, which is also caused by}

the interaction of capillarity and compressibility, as well as the nonlinearity of capillary phenomena. This effect is described by the second term in (2.10)-c : $p_{c}^{\mathcal{M}}-p_{c}^{\mathcal{F}}=\frac{\tau^{\mathrm{cc}}}{\beta_{\mathrm{o}}^{\mathcal{M}}} \frac{\partial \mathcal{S}_{w}^{\mathcal{M}}}{\partial t}$

5. Approach to description of highly compressible porous media. The results obtained concern weakly compressible porous media, so that the compressibility coefficients of pores are included in the structure of the original equations, but the porosity and permeability are considered to be very weakly dependent on pressure. How can this condition of compressibility be removed? Assume that the medium can contract strongly. Suppose that in this case the phase permeabilities and capillary pressure curves of blocks and fracture do not change. Then there is every reason to believe that the strong compressibility of the medium will not affect the structure of the asymptotic expansions in its first terms, which means that the obtained macroscopic equations (2.10) will not formally change. However, the porosity $\phi^{\mathcal{F}}$ and $\phi^{\mathcal{M}}$ and permeability $K^{\mathcal{F}}$ and $K^{\mathcal{M}}$ are now dependent on pressure. Then the effective permeability tensor (2.11), (2.12) also depends on the pressure through the permeability of the fractures $K^{\mathcal{F}}$. The delay times (2.13) will also depend on the pressure through the porosity of the blocks $\phi^{\mathcal{M}}$ and the permeability of the blocks $K^{\mathcal{M}}$, which is part of the cell problem (2.14). Moreover, since the pressures in water and oil are different, permeability $K^{\mathcal{F}}$ and $K^{\mathcal{M}}$ and porosity $\phi^{\mathcal{M}}$ behave differently in pores occupied by water and oil. This can be taken into account if we consider them to be dependent on pressures averaged over phases: 


$$
\mathcal{P}^{\mathcal{F}} \equiv \mathcal{P}_{w}^{\mathcal{F}} \mathcal{S}_{w}^{\mathcal{F}}+\mathcal{P}_{o}^{\mathcal{F}}\left(1-\mathcal{S}_{w}^{\mathcal{F}}\right) \quad \text { И } \quad \mathcal{P}^{\mathcal{M}} \equiv \mathcal{P}_{w}^{\mathcal{M}} \mathcal{S}_{w}^{\mathcal{M}}+\mathcal{P}_{o}^{\mathcal{M}}\left(1-\mathcal{S}_{w}^{\mathcal{M}}\right)
$$

Thus, a situation arises in which the cell problems (2.12) and (2.14) depend on macroscopic variables $\mathcal{P}_{w}^{\mathcal{M}}, \mathcal{P}_{w}^{\mathcal{F}}, \mathcal{P}_{o}^{\mathcal{F}}, \mathcal{P}_{o}^{\mathcal{F}}, \mathcal{S}_{w}^{\mathcal{M}}, \mathcal{S}_{w}^{\mathcal{F}}$, which is a typical case of an incompletely averaged model. Although it can be used for qualitative analysis of the process, but it is no simpler than the initial non-averaged system of equations for numerical calculations.

The situation changes if the cell problems can be solved analytically. Then macroscopic pressures and saturations will arise in the structure of effective permeability and relaxation times in explicit form. This can be done for simple geometry of the block $Y^{\mathcal{M}}$. For example, for spherical blocks of radius $R$, the cell problem (2.14) has an exact analytical solution, and the cell problem (2.12) has an approximate solution obtained by the method of potential flow [20]:

$$
\varphi(y)=\frac{R^{2}-r^{2}}{6 K^{\mathcal{M}}}, \quad \psi_{k}(y) \approx \frac{y_{k}\left[2+\left(\frac{R}{r}\right)^{3}\right]}{2+\theta}-y_{k} \quad(k=1,2,3) ; \quad r \equiv \sqrt{y_{1}^{2}+y_{2}^{2}+y_{3}^{2}}
$$

where $R=l\left(\frac{3 \theta}{4 \pi}\right)^{\frac{1}{3}}$, and $l$ is the length (with dimension) of a single period of medium heterogeneity. Then it is possible to obtain:

$$
\langle\varphi\rangle_{\mathcal{M}}=\frac{l^{2}}{15 K^{\mathcal{M}}}\left(\frac{3 \theta}{4 \pi}\right)^{\frac{2}{3}}, \quad \mathbb{K} \approx 2 K^{\mathcal{F}} \frac{1-\theta}{2+\theta}
$$

These relationships should be supplemented by the compressibility law written with respect to permeability $K^{\mathcal{F}}$ and $K^{\mathcal{M}}$, for example, by an exponential law similar to (1.1):

$$
K^{\mathcal{M}}=K^{\mathcal{M}, 0} e^{\tilde{\beta}_{K}^{\mathcal{M}}\left(\mathcal{P}^{\mathcal{M}}-P^{0}\right)}, \quad K^{\mathcal{F}}=K^{\mathcal{F}, 0} e^{\tilde{\beta}_{K}^{\mathcal{F}}\left(\mathcal{P}^{\mathcal{F}}-P^{0}\right)}
$$


where $\tilde{\beta}_{K}^{\gamma}$ and $\tilde{\beta}_{K}^{\gamma}$ are the coefficients of compressibility with respect to permeability; the pressures $\mathcal{P}^{\mathcal{F}}$ and $\mathcal{P}^{\mathcal{M}}$ are determined in (5.1); the superscript «zero» means a characteristic value.

Now, model (2.10) with relationships (5.2), (5.3) and (5.1) is completely averaged, but substantially nonlinear with respect to pressure.

In particular, for the single-phase case, we obtain a closed nonlinear flow system of a (strongly) compressible fluid in a (strongly) compressible medium:

$$
\begin{aligned}
& \beta^{\mathcal{F}}(1-\theta) \phi^{\mathcal{F}}\left(\mathcal{P}^{\mathcal{F}}\right) \partial_{t} \mathcal{P}^{\mathcal{F}}-\partial_{x i}\left(\mathbb{K}\left(\mathcal{P}^{\mathcal{F}}\right) \partial_{x i} \mathcal{P}^{\mathcal{F}}\right)=\xi\left(\mathcal{P}^{\mathcal{F}}\right)\left(\mathcal{P}^{\mathcal{M}}-\mathcal{P}^{\mathcal{F}}\right) \\
& \mathcal{P}^{\mathcal{M}}-\mathcal{P}^{\mathcal{F}}=-\tau^{\operatorname{comp}}\left(\mathcal{P}^{\mathcal{M}}\right) \frac{\partial \mathcal{P}^{\mathcal{M}}}{\partial t} \\
& \xi=\frac{15 \theta^{1 / 3}\left(\frac{4 \pi}{3}\right)^{2 / 3}}{\sqrt{\varepsilon} l^{2}} K^{\mathcal{M}}\left(\mathcal{P}^{\mathcal{M}}\right), \\
& \tau^{\text {comp }}=\frac{\sqrt{\varepsilon}}{15} \beta^{\mathcal{M}} l^{2} \mu\left(\frac{3 \theta}{4 \pi}\right)^{2 / 3} \frac{1-\theta}{2+\theta} K^{\mathcal{F}}\left(\mathcal{P}^{\mathcal{F}}\right) \\
& K^{\mathcal{M}}\left(\mathcal{P}^{\mathcal{M}}\right)
\end{aligned}
$$

where $\beta^{\mathcal{F}}, \beta^{\mathcal{M}}, \theta, \mu, l, \varepsilon$ are the constant values, and the functions $\phi^{\mathcal{M}}\left(\mathcal{P}^{\mathcal{M}}\right), \phi^{\mathcal{F}}\left(\mathcal{P}^{\mathcal{F}}\right), K^{\mathcal{M}}\left(\mathcal{P}^{\mathcal{M}}\right), K^{\mathcal{F}}\left(\mathcal{P}^{\mathcal{F}}\right)$ are described by formulae (5.3).

Conclusion. The averaged model (2.10) is of theoretical interest. Firstly, it is completely averaged, that is, it has no microscopic variables, and the cell problems (2.12), (2.14) do not contain macroscopic variables, therefore they are solved only once. Secondly, the model predicts and describes several delay mechanisms, some of which are nontrivial. One could intuitively expect that the introduction of compressibility into a two-phase system will lead to a double delay: capillary 
(typical for incompressible two-phase flows) and depression (typical for single-phase compressible flows). However, we have shown additional cross mechanisms of phase redistribution caused by phase extrusion and nonlinear extrusion, which, in turn, are non-trivially dependent on the asymmetry or symmetry of the compressibility process with respect to two phases.

The practical application of this model becomes important in cases of highly unsteady processes, when compressibility plays a significant role in the propagation of perturbations. Various memory effects are especially pronounced in processes with a variable direction of evolution. For example, phenomena in underground gas storages in which gas injection into an aquifer alternates with its withdrawal. The accumulation of the history of pressure and saturation oscillations in such processes can lead to a significant delay and a complete discrepancy in the qualitative behavior of the process compared to the process without memory.

We considered only the case of the exponential compressibility law, which is applicable to liquids and solids, but not to gases. Therefore, the applicability of this model for a gas-liquid system is possible only for the case of highly compressed gas at high pressures, whose properties are close to liquid.

This work was supported by the Scientific Committee of the Ministry of Education and Science of the Republic of Kazakhstan: grant $\mathrm{n}^{\circ}$ AP05132680

\section{REFERENCES}

1. Ait Mahiout L., Amaziane B., Mokrane A., Pankratov L. Homogenization of immiscible compressible twophase flow in double porosity media // Electronic J. Differential Equations. 2016. V. 52. P. 1-28. 
2. Allaire G. Homogenization and two-scale convergence // SIAM J. Math. Anal. 1992. V. 28. P. 1482-1518.

3. Amaziane B., Jurak M., Pankratov L., Vrbaski A. Some remarks on the homogenization of immiscible incompressible two-phase flow in double porosity media. // Discrete and Continuous Dynamical Systems, Ser. B. 2018. V. 23. № 2. P. 629-665.

4. Amaziane B., Milisic J.P., Panfilov M., and Pankratov L. Generalized nonequilibrium capillary relations for two-phase flow through heterogeneous media // Physical Review E. 2012. V. 85, P. 016304.

5. Amaziane B., and Pankratov L. Homogenization of a model for water-gas flow through double-porosity media // Mathematical Methods in the Applied Sciences. 2016. V. 39. P. 425-451.

6. Amaziane B., Pankratov L., Jurak M., and Vrbaski A. A fully homogenized model for incompressible two-phase flow in double porosity media // Applicable analysis. 2015. April

7. Arbogast T., Douglas J., and Hornung U. Derivation of the double porosity model of single phase flow via homogenization theory // SIAM J. Math. Anal. 1990. V. 21. № 4. P. 823-836.

8. Arbogast T. A simplified dual porosity model for two-phase flow // Computational Methods in Water Resources IX, Vol. 2: Mathematical Modeling in Water Resources. Eds T.F. Russell et al. Southampton: Computational Mechanics Publications. 1992 
9. Barenblatt G.I., Zheltov Yu.P., Kochina I.N. On basic concepts of the theory of homogeneousfluids seepage in fractured rocks // Prikl. Mat. Mekh. 1960. V. 24. № 5. P. 852-864

10. Bourgeat A., Luckhaus S., Mikelic A. Convergence of the homogenization process for a double-porosity model of immiscible two-phase flow // SIAM J. Math. Anal. 1966. V. 27. № 6. P.1520-1543.

11. Bourgeat A., Panfilov M. Effective two-phase flow through highly heterogeneous porous media // Computational Geosciences. 1998. V. 2. P. $191-215$.

12. Hussein M. Multiphase flow simulations in heterogeneous fractured media through hybrid grid method // AIP Conference Proceedings. 2013. № 1558. P. 2048.

13. Jafari I, Masihi M., Nasiri Zarandi M. Numerical simulation of countercurrent spontaneous imbibition in water-wet fractured porous media: Influences of water injection velocity, fracture aperture, and 826 grains geometry // Phys. Fluids. 2017. V. 29. № 11. P. 113305.

14. Jafari I., Masihi M., Nasiri Zarandi M. Experimental study on imbibition displacement mechanisms of two-phase fluid using micromodel: Fracture network, distribution of pore size, and matrix construction // Phys. Fluids. 2017. V. 29. № 11. P. 122004.

15. Khoshkalam Y., Khosravi M., Rostami B. Visual investigation of viscous cross-flow during foam injection in a matrix-fracture system // Phys. Fluids. 2019. V. 31. P. 023102. 
16. Li H., Guo H., Yang Z. et al. Evaluation of oil production potential in fractured porous media // Phys. Fluids. 2019. V. 31. P. 052104.

17. Meirmanov A.M. Mathematical Models for Poroelastic Flows, Atlantis Studies in Differential Equations, v.1. // Amsterdam - Paris - Beijing: Atlantis Press, 2014. 308 p.

18. Meirmanov A.M. Reiterated homogenization in the problems of filtration of underground liquids // Scientific notes of the Belgorod State University. Ser. Mathematics-Physics, 2012

19. Nikolaevsky V.N. Mechanics of porous and fractured media. Moscow: Nedra, 1984. 233 p. ]

20. Panfilov $M$. Macroscale models of flow through highly heterogeneous porous media // Dordrecht: Kluwer Academic Publishers, 2000. 363 p.

21. Rokhforouz M.R., Akhlaghi Amiri H.A. Phase-field simulation of countercurrent spontaneous imbibition in a fractured heterogeneous porous medium // Phys. Fluids. 2017. V. 29. P. 062104.

22. Saedi B., Ayatollahi S., Masihi M. Free fall and controlled gravity drainage processes in fractured porous media: Laboratory and modelling investigation // Can. J. Chem. Eng. 2015. V. 93. P. 2286.

23. Yao C.C., Yan P.Y. A diffuse interface approach to injection-driven flow of different miscibility in heterogeneous porous media // Phys. Fluids. 2015. V. 27, № 8. P. 083101.

24. Yeh L.M. Homogenization of two-phase flow in fractured media // Math. Models Methods Appl. Sci. 2006. V. 16. P. 1627-1651. 
25. Scheidegger A.E. The Physics of Flow through Porous Media. 3rd Edition // Toronto: University of Toronto Press, 1974. 372 p. 